\title{
Development of genome-wide informative simple sequence repeat markers for large-scale genotyping applications in chickpea and development of web resource
}

\author{
Swarup K. Parida, Mohit Verma, Santosh K. Yadav, Supriya Ambawat, Shouvik Das, \\ Rohini Garg and Mukesh Jain*
}

Functional and Applied Genomics Laboratory, National Institute of Plant Genome Research, New Delhi, India

\section{OPEN ACCESS}

Edited by:

Frédéric Marsolais,

Agriculture and Agri-Food Canada,

Canada

Reviewed by:

Teresa Millan,

Universidad de Córdoba, Spain

Pawan L. Kulwal,

Mahatma Phule Agricultural

University, India

*Correspondence:

Mukesh Jain,

Functional and Applied Genomics Laboratory, National Institute of Plant

Genome Research, Aruna Asaf All

Marg, New Delhi 110067, India

mjain@nipgr.ac.in

Specialty section:

This article was submitted to

Plant Genetics and Genomics,

a section of the journal

Frontiers in Plant Science

Received: 16 January 2015

Accepted: 03 August 2015

Published: 21 August 2015

Citation:

Parida SK, Verma M, Yadav SK,

Ambawat S, Das S, Garg R

and Jain M (2015) Development of genome-wide informative simple

sequence repeat markers

for large-scale genotyping applications in chickpea and development of web resource.

Front. Plant Sci. 6:645

doi: 10.3389/fpls.2015.00645
Development of informative polymorphic simple sequence repeat (SSR) markers at a genome-wide scale is essential for efficient large-scale genotyping applications. We identified genome-wide 1835 SSRs showing polymorphism between desi and kabuli chickpea. A total of 1470 polymorphic SSR markers from diverse coding and noncoding regions of the chickpea genome were developed. These physically mapped SSR markers exhibited robust amplification efficiency (73.9\%) and high intra- and inter-specific polymorphic potential (63.5\%), thereby suggesting their immense use in various genomics-assisted breeding applications. The SSR markers particularly derived from intergenic and intronic sequences revealed high polymorphic potential. Using the mapped SSR markers, a wider functional molecular diversity (16-94\%, mean: 68\%), and parentage- and cultivar-specific admixed domestication pattern and phylogenetic relationships in a structured population of desi and kabuli chickpea genotypes was evident. The intra-specific polymorphism (47.6\%) and functional molecular diversity (65\%) potential of polymorphic SSR markers developed in our study is much higher than that of previous documentations. Finally, we have developed a user-friendly web resource, Chickpea Microsatellite Database (CMsDB; http://www.nipgr.res.in/CMsDB. $\mathrm{html})$, which provides public access to the data and results reported in this study. The developed informative SSR markers can serve as a resource for various genotyping applications, including genetic enhancement studies in chickpea.

Keywords: chickpea, genotyping, molecular diversity, polymorphism, population structure, simple sequence repeat

\section{Introduction}

In recent years, a significant progress has been made concerning the development of numerous genomic and transcript-derived simple sequence repeat (SSR) and single nucleotide polymorphism (SNP) markers at a genome-wide scale and their deployment in multi-dimensional genomicsassisted breeding applications in chickpea (Winter et al., 2000; Abbo et al., 2005; Millan et al., 2010; Nayak et al., 2010; Gujaria et al., 2011; Thudi et al., 2011; Gaur et al., 2012; Hiremath et al., 2012; Roorkiwal et al., 2013; Deokar et al., 2014; Jaganathan et al., 2014). This suggests 
that development, large-scale validation and use of functionally relevant informative sequence-based robust genetic markers revealing high intra-specific polymorphic potential are preferred in marker-assisted genetic enhancement studies of chickpea. Due to many inherent desirable genetic attributes, including abundance, co-dominant inheritance, reproducibility, multiallelic nature and simpler genotyping potential (gel-based assay), SSRs have been considered as marker of choice in crop plants, including chickpea (Varshney et al., 2005, 2014; Parida et al., 2006; Sethy et al., 2006; Radhika et al., 2007; Upadhyaya et al., 2008; Nayak et al., 2010; Anuradha et al., 2011; Bharadwaj et al., 2011; Gujaria et al., 2011; Vadez et al., 2012).

Length polymorphism of SSR markers in the coding sequence (CDS) and non-CDS components $\left[5^{\prime}\right.$-untranslated regions ( $5^{\prime}$-UTRs), introns and $3^{\prime}$-untranslated regions ( $3^{\prime}$-UTRs)] of genes are known to affect transcription and translation, and may have significant consequences on gene function ( $\mathrm{Li}$ et al., 2004). The expansion and contraction of SSR repeats in the $5^{\prime}-$ UTRs, for instance, have significance in regulating many traits (Dresselhaus et al., 1999; Bao et al., 2002; Zhang et al., 2006). The length polymorphism in the functional domain of transcription factor genes, and alteration of secondary structure of proteins and functional domain sites have been proposed to control seed weight/seed size in chickpea (Kujur et al., 2013). These studies have suggested the utility of coding and non-CDS-based SSR markers for rapidly establishing marker-trait linkages and identifying genes/QTLs for many useful agronomic traits in crop plants. The SSR markers particularly derived from the nonCDS components of genes with moderate selection pressure are expected to reveal high intra-specific polymorphism in contrast to highly constrained CDS-based markers (Cho et al., 2000; Chabane et al., 2005; Parida et al., 2010; Kujur et al., 2013).

The utility of genome-wide identification of polymorphic SSR markers by comparing the genomic or transcript sequences between indica and japonica rice (Grover et al., 2007; Zhang et al., 2007), and Setaria italica and S. viridis (Zhang et al., 2014) as well as among chickpea genotypes (Agarwal et al., 2012; Jhanwar et al., 2012) in large-scale genotyping applications has been well demonstrated. A large chickpea genome ( $\sim 40 \mathrm{Mb})$ with narrow genetic base requires a huge number of such functionally relevant polymorphic SSR markers at a genomewide scale for various applications in structural, functional, and applied genomics. With the availability of draft genome sequences of desi and kabuli chickpea (Jain et al., 2013; Varshney et al., 2013), it is now possible to mine informative SSR markers from coding and non-CDS components of genes in the two chickpea genomes. Recently, SSR markers identified from the kabuli genome have been made publicly accessible via a web resource, Cicer arietinum Microsatellite Database (CicArMiSatDB; Doddamani et al., 2014). The informativeness of such genome-wide SSR markers can further be enriched by identifying a subset of markers showing polymorphism between desi and kabuli chickpea in different sequence components of annotated genes.

Keeping all above in view, the present study was undertaken to mine and characterize SSR repeat-motifs in different coding and non-CDS components, and intergenic regions between desi and kabuli chickpea genomes and develop genomewide polymorphic SSR markers. The developed markers were evaluated to determine their amplification and polymorphic potential, and assessment of functional molecular diversity and population genetic structure among desi and kabuli chickpea genotypes. In addition, we have developed an easy-to-use web resource for public access of the SSR data generated in this study. The development of informative markers would further expedite the process of construction of high resolution genetic map, and identification and mapping of genes/QTLs regulating important agronomic traits for genetic improvement of chickpea.

\section{Materials and Methods}

\section{Discovery of SSRs in Chickpea Genomes}

The desi (Cicer arietinum L. cv. ICC4958; Jain et al., 2013) and kabuli (C. arietinum L. cv. CDC Frontier; Varshney et al., 2013) chickpea genomes were obtained from Chickpea Genome Analysis Project ${ }^{1}$ and International Chickpea Genetics $\&$ Genomics Consortium ${ }^{2}$, respectively. These sequences were mined for SSRs using MISA (MIcroSAtellite ${ }^{3}$ ) following the criteria (at least six repeats of di-nucleotides and five repeats of tri- to hexa-nucleotides) as described earlier (Garg et al., 2011; Jhanwar et al., 2012). The perfect SSRs were further classified into hypervariable class I ( $\geq 20 \mathrm{bp}$ ) and potentially variable class II (12-20 bp) types according to length of repeatmotifs. The structural annotation of identified SSRs in different coding (CDS) and non-coding (5'-UTRs, introns and $3^{\prime}$-UTRs) sequence components of chickpea genes, and upstream regions (1000 bp) and intergenic regions was performed based on the available genome annotation. The putative function of SSRs containing gene sequences was determined based on their available functional annotation information.

\section{Development of Gene-Derived Polymorphic SSR Markers in Chickpea}

The SSRs showing polymorphism between desi and kabuli chickpea were identified using the approach described by Zhang et al. (2007). In the first step, 250 bp flanking sequences on each side of the identified SSR motifs were retrieved from both the genomes and searched against each other via BLASTN. Only the sequences showing unique hit in the reciprocal BLAST results with an $E$-value cut-off of $\leq 1 \mathrm{e}-40$ were retained. This resulted in the identification of 13327 orhtologous SSR loci in the two genomes. Further, we used custom designed perl script to identify and characterize the polymorphic SSR loci between desi and kabuli chickpea based on difference in number of repeat-units present, as described by Jhanwar et al. (2012). The forward and reverse primers from the genomic sequences of ICC 4958 flanking polymorphic SSR repeat-motifs were designed using the Primer3 tool at default parameters. The polymorphic SSR markers were physically mapped on eight

\footnotetext{
${ }^{1}$ http://nipgr.res.in/CGAP/home.php

${ }^{2}$ http://www.icrisat.org/gt-bt/ICGGC/GenomeManuscript.htm

${ }^{3}$ http://pgrc.ipk-gatersleben.de/misa/
} 
chromosome pseudomolecules of chickpea according to their genomic location.

\section{Evaluation of Amplification Efficiency and Polymorphic Potential}

A total of 341 selected polymorphic SSRs (showing $\geq 4$ bp fragment length polymorphism between desi and kabuli chickpea) present in the coding and non-CDS components of genes, and intergenic regions were amplified via PCR using genomic DNA of 31 desi and 15 kabuli chickpea genotypes to evaluate their amplification and polymorphic potential. These desi and kabuli genotypes [collected from Indian Agricultural Research Institute (IARI), New Delhi and International Crops Research Institute for the SemiArid Tropics (ICRISAT), Hyderabad] with diverse useful yield contributing and stress tolerance traits, have been primarily utilized as contrasting parents in various cross-breeding varietal improvement programs for developing improved cultivars of chickpea in India. The touchdown thermal cycling profiling and standard constituents used for PCR amplification were as described previously (Agarwal et al., 2012; Jhanwar et al., 2012). The PCR products amplified by each SSR marker in the 46 chickpea genotypes were resolved on $3.5 \%$ metaphor agarose gel and their allele size and fragment length polymorphism was determined. The genotyping information of SSR markers was used to estimate the number of polymorphic alleles per marker locus, percent polymorphism and polymorphism information content (PIC) among genotypes using PowerMarker v3.51 (Liu and Muse, 2005). The polymorphic SSRs were positioned on the chickpea chromosomes according to their genomic coordinates using MapChart (v2.2).

\section{Functional Molecular Diversity and Population Structure Analysis}

A total of 160 informative SSR markers were used for determining functional molecular diversity and establishing phylogenetic relationships among chickpea genotypes based on Nei's genetic distance (Nei et al., 1983) by neighbor-joining (NJ) method (with 1,000 bootstrap replicates) using PowerMarker v3.51 and unrooted phylogenetic tree was constructed. For assessment of population structure, the SSR marker genotyping data were analyzed in STRUCTURE (Pritchard et al., 2000) with burn-in of 100000 iterations and run length of 1000000, following the method described by Kujur et al. (2013). The genetic variability $\left(F_{\mathrm{ST}}\right)$ and degree of admixture within and between population groups at optimal $K$ (population number) value was determined.

\section{Construction of Chickpea Microsatellite Database}

We developed a user-friendly web resource, Chickpea Microsatellite Database (CMsDB), to provide browsable access to the SSR data. The web pages of CMsDB have been written using Perl-CGI on the Apache Tomcat (version 5.5.29) Web server application. The information regarding SSRs, their flanking sequences and primer details are cataloged in the MySql server (version 5.0.77). The database is currently hosted on Sun Workstation running CentOs (version 5.4) Linux operating system with two Intel Xeon quad core processors and $12 \mathrm{~GB}$ of random access memory. The database is compatible with various browsers like Internet Explorer, Mozilla Firefox, and Google Chrome.

\section{Results}

\section{Frequency and Distribution of SSRs in desi and kabuli Chickpea Genomes}

A total of 519.8 and $522.3 \mathrm{Mb}$ sequences of desi and kabuli chickpea genomes, respectively, were utilized for mining and characterization of SSR motifs. Based on these analyses, 74941 and 81845 perfect SSRs (excluding mono-nucleotides) were identified in desi and kabuli chickpea with an average density of $0.144 \mathrm{SSR} / \mathrm{kb}$ and $0.157 \mathrm{SSR} / \mathrm{kb}$, respectively (Table 1). The overall frequency of compound SSRs identified in desi (2563, $0.005 \mathrm{SSRs} / \mathrm{kb})$ and kabuli (2470, $0.004 \mathrm{SSRs} / \mathrm{kb})$ chickpea was almost comparable with each other. In both the genomes, di-nucleotide repeat-motifs (desi: 41457, 55.3\% and kabuli: 47127 , $57.6 \%$ ) were most prevalent followed by tri- and tetra-nucleotides (Table 1). In terms of proportion of total number of SSRs identified, the long hyper-variable class I repeats varied from $49.8 \%$ (40789) in kabuli to 50.3\% (37737) in desi chickpea. The class I and class II di-nucleotide repeat-motifs were present in maximum fraction varying from 49.1 (18537) to $62.3 \%$ (25568) in desi and kabuli chickpea (Figure 1A). Next, the tri-nucleotide SSR repeat-motifs were most abundant (varied from 34.2 to $38.4 \%$ ) in both class I and class II SSRs, while tetra-, penta-, and hexa-nucleotide motifs were completely absent in case of class II SSRs. The frequency of AT-rich di-nucleotide repeatmotifs (32354 SSRs in desi, $43.2 \%$ and 37977 SSRs in kabuli, 46.4\%) was maximum in both desi and kabuli chickpea followed by AAT/ATT-rich tri-nucleotide SSRs (21113 SSRs, $28.2 \%$ in desi and 22003, 26.9\% in kabuli) and AAAT/ATTT-rich tetranucleotide SSRs (3095, 4.1\% and 2924, 3.6\%) (Figure 1B). The structural annotation of identified SSRs in desi genome revealed their highest frequency in intergenic regions (64961

TABLE 1 | Summary of SSRs identified in desi and kabuli chickpea genomes.

\begin{tabular}{lll}
\hline Characteristic & Desi & Kabuli \\
\hline Total number of sequences examined & 181462 & 7135 \\
Total size (bp) of examined sequences & 519846222 & 522314610 \\
Total number of identified SSRs & 74941 & 81845 \\
Number of SSRs containing sequences & 18559 & 1769 \\
Number of SSRs present in compound & 2563 & 2470 \\
formation & & \\
Frequency of SSRs & 0.144 SSR/kb & 0.157 SSR/kb \\
Number (\%) of di-nucleotides & $41457(55.3)$ & $47127(57.6)$ \\
Number (\%) of tri-nucleotides & $28042(37.4)$ & $29442(36.0)$ \\
Number (\%) of tetra-nucleotides & $4098(5.5)$ & $3944(4.8)$ \\
Number (\%) of penta-nucleotides & $740(0.98)$ & $781(0.95)$ \\
Number (\%) of hexa-nucleotides & $604(0.81)$ & $551(0.67)$ \\
Number (\%) of class I SSRs & $37737(50.4)$ & $40789(49.8)$ \\
Number (\%) of class II SSRs & $37204(49.6)$ & $41056(50.2)$
\end{tabular}


A

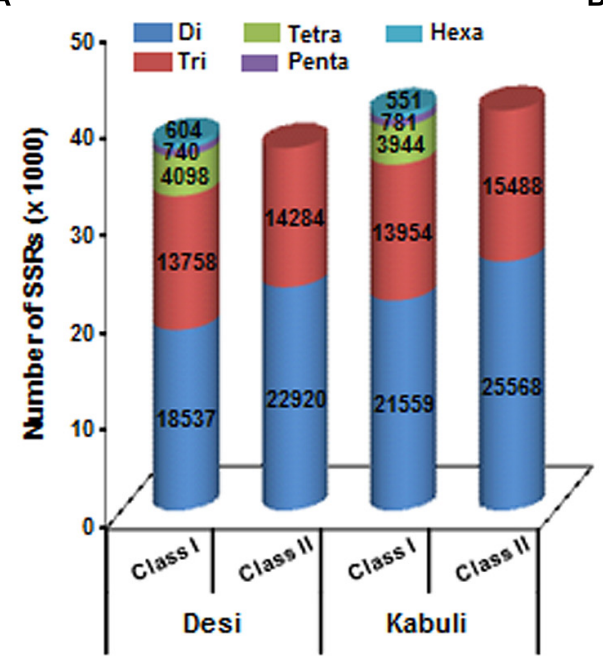

B

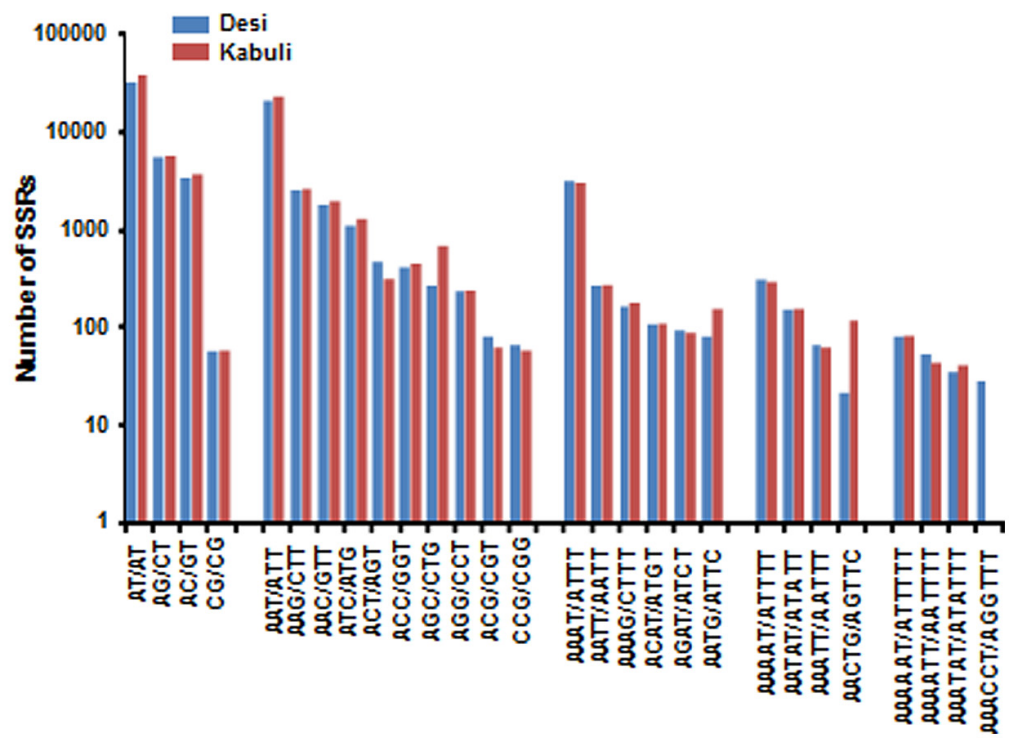

FIGURE 1 | Classification and frequency of simple sequence repeats (SSRs) identified in desi and kabuli chickpea genomes. (A) The bar graph displays the number of SSRs of different types in long hypervariable class I ( $\geq 20 \mathrm{nt}$ ) and potentially variable class II (12-20 nt). (B) Frequency of major SSR motifs of different classes identified in desi and kabuli genomes.

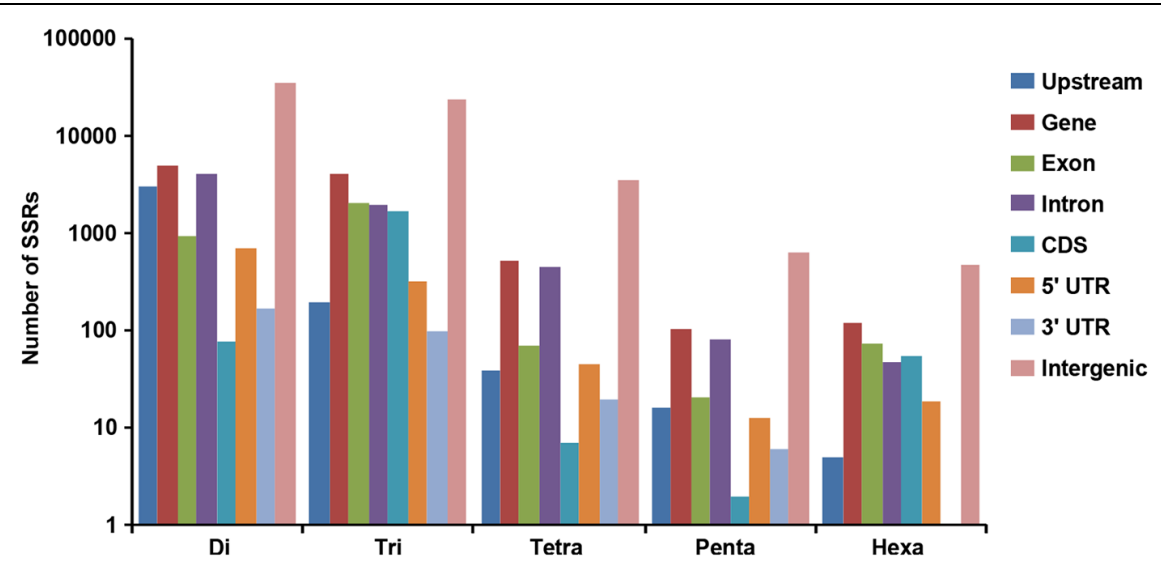

FIGURE 2 | Distribution of SSRs identified from desi and kabuli chickpea genomes in different regions of the chickpea genome. The bar graph displays the number of SSRs of different classes located in different genomic features (various gene components and intergenic regions) of the chickpea genome.

SSRs) followed by introns (6762), exons (3218), CDS (1816), and upstream regions (3289) (Figure 2). All five different classes of SSR repeat-motifs (di- to hexa-nucleotides) were predominant particularly in the intergenic regions as compared to various coding and non-CDS components of genes. However, within genes, the frequency of di- (4191), tetra- (462), and penta-nucleotide (84) SSR repeat-motifs were maximum in the intronic and upstream sequences, whereas tri- (2100) and hexanucleotide (76) motifs were abundant in the exons (Figure 2). Maximum number of tri-nucleotide SSR repeat-motifs was found in the CDS (1673 SSRs), whereas upstream regions (3029), and 5 '- (703) and 3'-UTRs (168) were rich in di-nucleotide repeatmotifs.

\section{Density and Relative Abundance of Polymorphic SSRs}

We identified 1835 genome-wide SSRs showing polymorphism between desi and kabuli chickpea based on their expansion/contraction of SSR repeat-length (ranged from 2 to $>20 \mathrm{bp}$; Figures $\mathbf{3 A}, \mathbf{B})$. Among the polymorphic SSRs obtained, the di-nucleotide repeat-motifs (1425 SSRs, 77.6\%) were most abundant followed by tri-nucleotide motifs (388, $21.1 \%$ ) (Figure 3B). With the increase of SSR repeat-length variation, the number of SSRs showing polymorphism decreased, which indicates inverse correlation between fragment length polymorphism and frequency of polymorphic SSRs identified in desi and kabuli chickpea (Figure 3A). Maximum number 
A

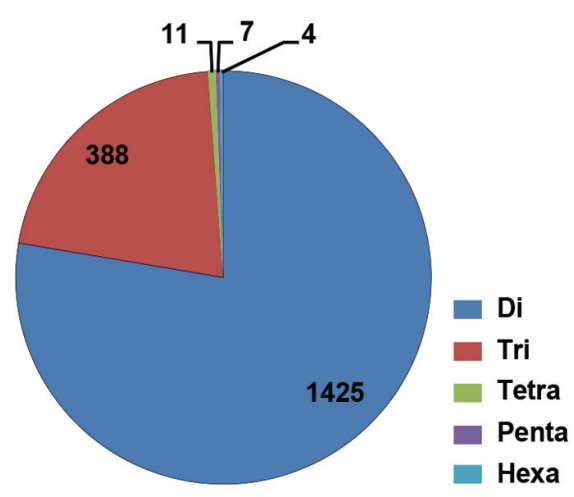

C

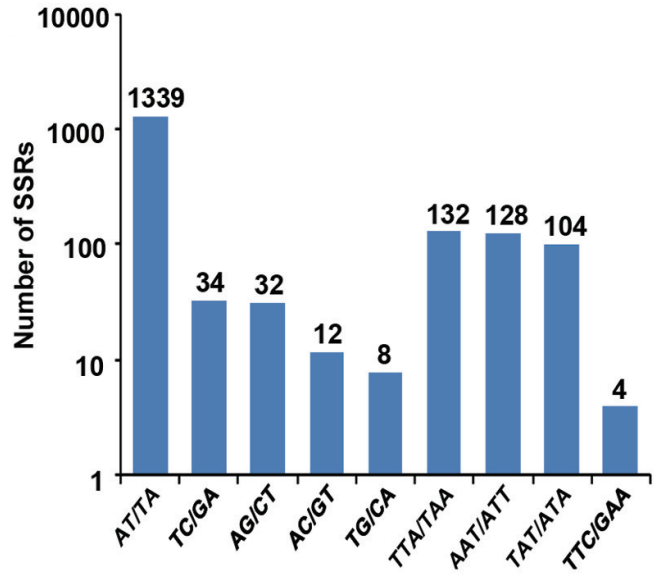

FIGURE 3 | Identification and analysis of polymorphic SSRs between desi and kabuli chickpea. (A) Distribution of polymorphic SSRs in different classes. (B) Number of SSRs with different motif length (2 to >20) differences.

D
B
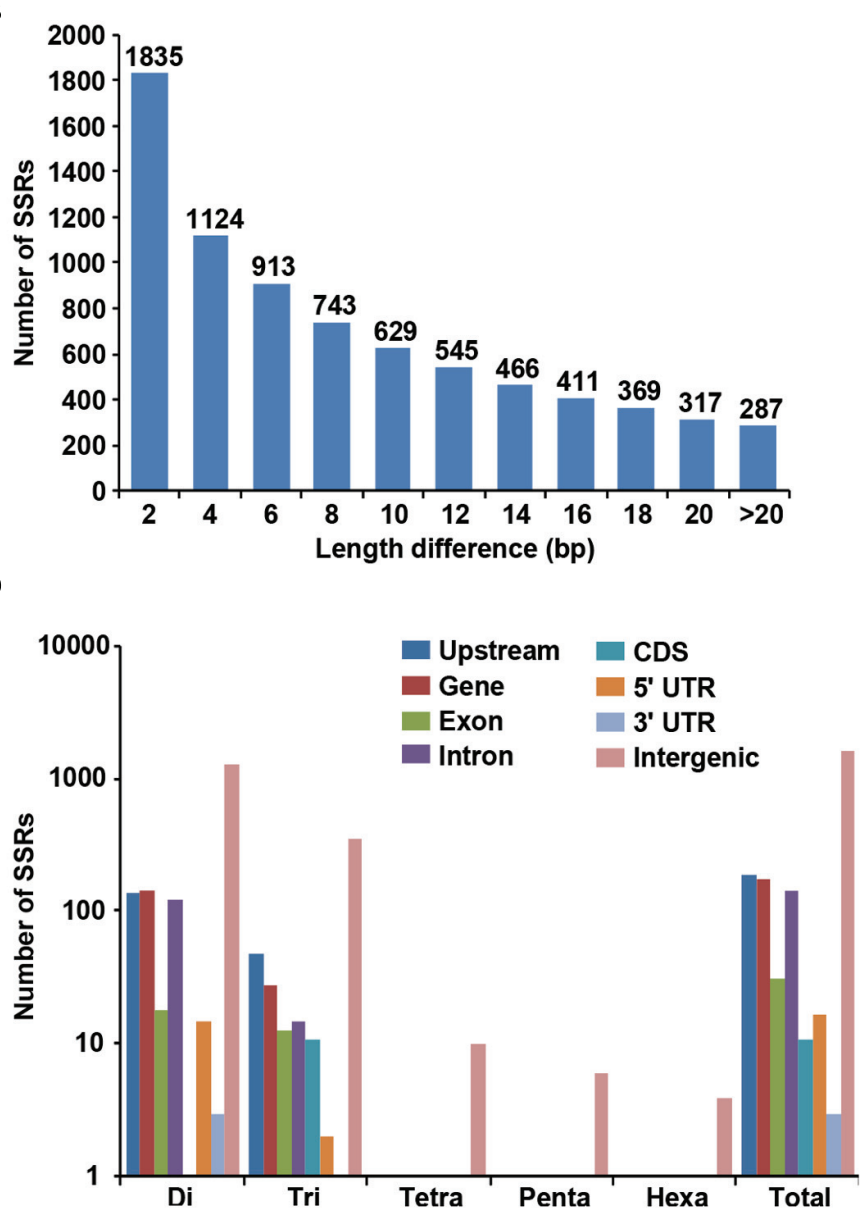

(C) Frequency of selected (most abundant) motifs in the polymorphic SSRs. (D) Distribution of polymorphic SSRs in in different genomic features (various gene components and intergenic regions). of polymorphic SSRs showed $\geq 2$-bp repeat-unit variation and 287 SSRs with $>20$-bp variation between desi and kabuli chickpea were identified. The AT/TA di-nucleotide repeats (1339, 73\%) were maximum followed by TTA/TAA (132, $7.2 \%)$, AAT/ATT $(128,6.8 \%)$, and TAT/ATA (104, 5.7\%) (Figure 3C). All the identified 1835 polymorphic SSRs were structurally annotated in intergenic regions, and coding and non-CDS components of genes. Maximum number of polymorphic SSR repeats were identified in the intergenic regions (1453 SSRs, 79.2\%), (Figure 3D). The polymorphic SSRs identified within genes included highest number in the upstream sequences $(190,10.4 \%)$ followed by introns (143, 7.8\%), exons $(31,1.7 \%)$ and minimum in $3^{\prime}$-UTRs $(3,0.16 \%)$ of genes. All classes of polymorphic SSR repeats including di- to hexa-nucleotide motifs were maximum in the intergenic sequences (Figure 3D). The di-nucleotide followed by tri-nucleotide SSR repeats were abundant in the upstream regions (139 di- and 49 tri-nucleotide) and intronic (126 di- and 15 tri-nucleotide) sequences of genes, whereas tri-nucleotide repeats were maximum in the coding regions.
About $85 \%$ of the polymorphic SSRs were present on the eight chickpea chromosomes, whereas other $15 \%$ were located on the scaffolds of kabuli chickpea genome (Supplementary Figure S1). A complete list of polymorphic SSRs along with their motifs and genomic location in both genomes is given in the Supplementary Table S1.

The higher abundance of polymorphic SSRs specifically in the upstream regulatory regions and introns of chickpea genes is consistent with the previous reports in rice and Arabidopsis (Fujimori et al., 2003; Lawson and Zhang, 2006; Parida et al., 2009). The most frequent occurrences of di- and tri-nucleotide SSR repeats in the upstream regulatory regions of genes are expected. These SSR repeats can possibly facilitate or abolish binding sites of regulatory proteins and thus regulate gene expression (Yu et al., 2002; Lawson and Zhang, 2006; Parida et al., 2009). The presence of AT-rich di-nucleotide SSRs in the introns of chickpea genes is also comparable to that observed in the earlier studies of cereal genomes (Temnykh et al., 2001; Lawson and Zhang, 2006; Parida et al., 2009). The genome-wide identification and characterization of SSRs 
including those showing polymorphism between desi and kabuli revealed non-random and biased distribution across various genomic components.

\section{Development and Annotation of Polymorphic SSR Markers}

The forward and reverse primers from the flanking sequences of polymorphic 1835 SSR repeat-motifs were designed. The structural annotation and organization of selected SSR repeats located in different components of genes are presented in the Figure 4. We could design primers for 1470 (80.1\%) polymorphic SSRs, which included 1151 (78.3\%) in the intergenic regions, $150(10.2 \%)$ in the upstream regulatory sequences, $125(8.5 \%)$ in the introns, $31(2.1 \%)$ in the exons, $17(1.2 \%)$ in the $5^{\prime}$-UTRs, $11(0.75 \%)$ in the CDS, and $3(0.20 \%)$ in the $3^{\prime}$-UTRs (Supplementary Table S1). At least $85 \%$ of these genome-wide unique SSR markers were mapped on the chickpea chromosome pseudomolecules (Supplementary Figure S1). Maximum number of markers were mapped on chromosome $4(224,15.2 \%)$ followed by chromosome 5 (206, 14\%), while minimum number of markers mapped on chromosome 8 (72, $4.9 \%)$.

\section{Amplification and Polymorphic Potential of Developed SSR Markers}

We utilized 341 SSR markers (revealing $\geq 4$-bp fragment length polymorphism between desi and kabuli chickpea) in total located in different components of genes (upstream, $5^{\prime}$ UTRs, CDS, introns and $3^{\prime}$-UTRs) and intergenic regions to evaluate their amplification efficiency as well as potential for detecting polymorphism among 31 desi and 15 kabuli chickpea genotypes (Supplementary Table S3). Two hundred fifty-two of the 341 markers gave successful PCR amplification in all 46 chickpea genotypes with an amplification success rate of
73.9\% (Supplementary Table S2). One hundred sixty (63.5\%) of 252 amplified markers showed polymorphism in at least two combinations of chickpea genotypes (Figure 5A). It included 130 (73.9\%, mean PIC: 0.76$)$ of 176 class I and $30(39.5 \%$, 0.69 ) of 76 class II SSR markers. The remaining 92 (36.5\%) markers exhibited monomorphic amplification among chickpea genotypes used. A total number of 764 alleles were amplified by 160 polymorphic SSR markers with a mean allele number of 4.8. The number of alleles amplified per locus varied from 2 to 12 . The PIC ranged from 0.23 to 0.86 with an average of 0.75 , while gene diversity varied from 0.25 to 0.89 with a mean of 0.77 (Supplementary Table S2). The polymorphic potential of markers in different sequence components of genes and intergenic regions was analyzed in detail based on the percent polymorphism, PIC and polymorphic alleles amplified among chickpea genotypes. We were able to detect polymorphism in 55 (62.5\%, allele number from 2-9 and PIC 0.74) of 88 markers derived from the different coding and non-CDS components of genes between desi and kabuli chickpea. The remaining 105 (64\%, 2-12 and 0.63) of 164 markers derived from intergenic regions also showed polymorphism between the two chickpea types (Supplementary Table S2). Within genes, maximum potential of polymorphism was detected by the markers developed from intronic sequences (28 of 38 markers, $73.7 \%$, allele number 2-9 and PIC 0.74 ) followed by $3^{\prime}$-UTRs ( 5 of 8 markers, $62.5 \%, 2-$ 4 and 0.66 ), upstream (19 of 35 markers, $54.3 \%, 2-5$ and 0.76 ) and $5^{\prime}$-UTRs ( 2 of 6 markers, 33.3\%, 4-5 and 0.62) of genes (Supplementary Table S2, Figure 5A). Remarkably, 120 (47.6\%, $2-10$ and 0.62$)$ and 107 (42.5\%, 2-8 and 0.57) markers revealed polymorphism within desi and kabuli chickpea genotypes too, respectively. Overall, eight different representative allele types were detected based on fragment length polymorphism patterns of all the 160 SSR markers in 46 desi and kabuli genotypes (Figure 5B).

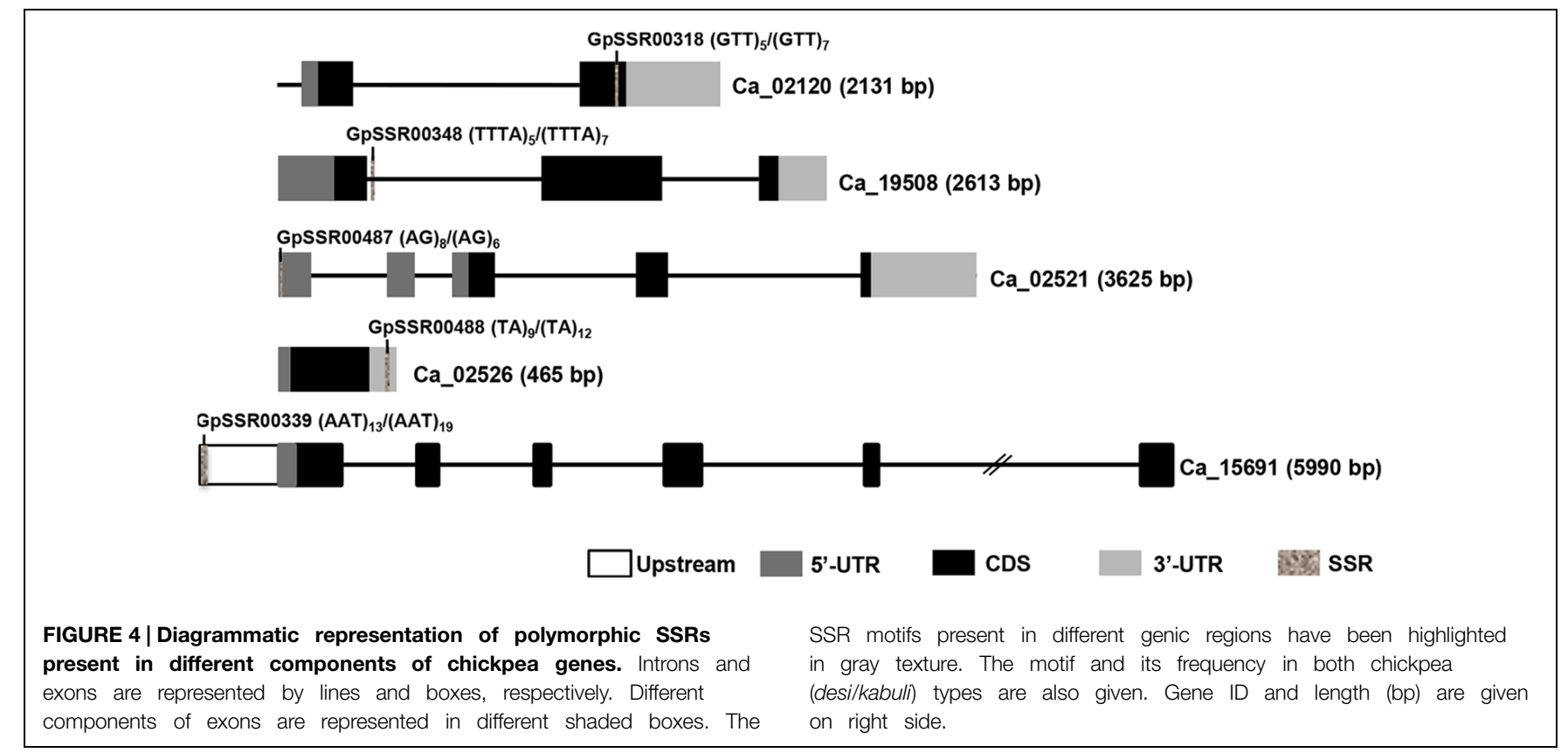




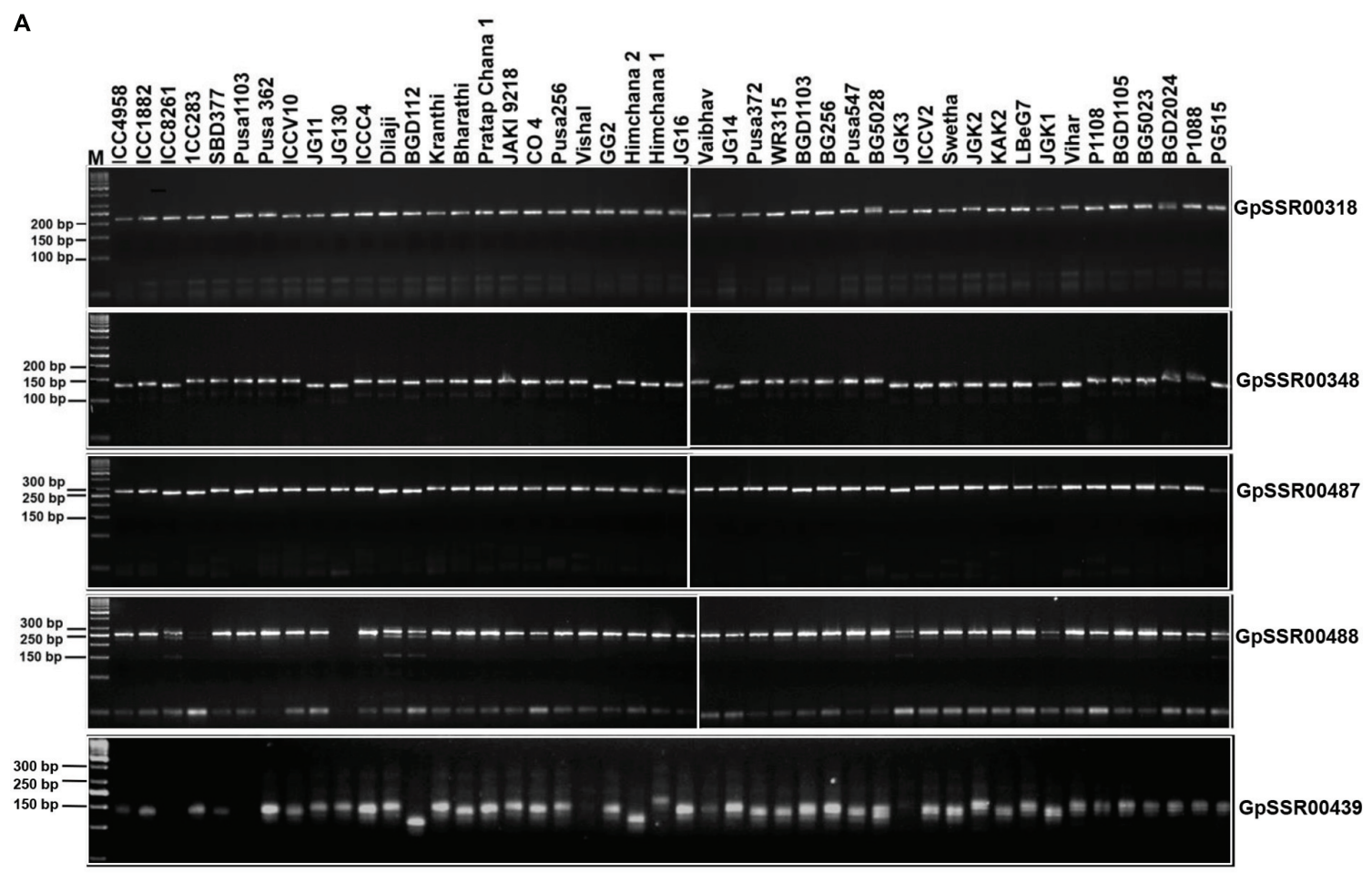

B

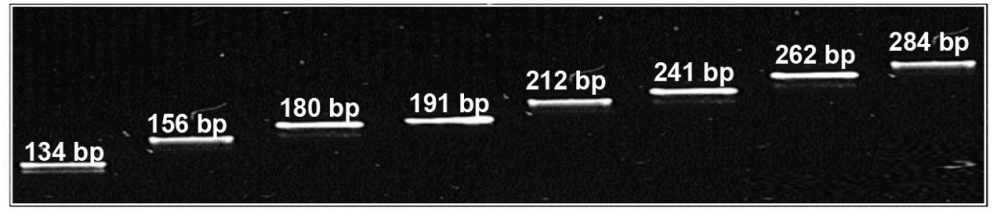

FIGURE 5 | Validation of amplification and polymorphic potential of selected SSRs and allelic variations in chickpea genotypes.

(A) Polymerase chain reaction based validation of amplification and polymorphic potential of selected SSRs in chickpea genotypes. Only five selected examples of SSRs have been represented. Representative gels showing PCR amplification of polymorphic SSRs (labeled on right side) validating the length polymorphism between desi and kabuli chickpea genotypes (genotype details are available in Supplementary Table S3). M, 50 bp DNA ladder as size standard. (B) Eight different representative allele types identified based on the fragment length polymorphism pattern of 160 SSR markers across desi and kabuli chickpea genotypes. The SSR marker-alleles are illustrated according to their lower to higher fragment size (bp).

\section{Functional Molecular Diversity and Population Structure Among desi and kabuli Chickpea}

The pair-wise distance matrix among 46 desi and kabuli chickpea genotypes based on genotyping information of 160 validated polymorphic SSR markers revealed a broad range of genetic distance that varied from 0.16 (kabuli cv. BGD1105 - kabuli cv. Pusa1088) to 0.94 (desi cv. Vishal - desi cv. ICC4958) with an average of 0.68 . Maximum average genetic distance was observed particularly among the accessions belonging to desi chickpea (0.65) in contrast to that detected within kabuli (0.57). The phylogenetic relationship among 31 desi and 15 kabuli chickpea genotypes has been depicted in an unrooted dendrogram (Figure 6). This set of informative genome-wide physically mapped SSR markers (160) clearly discriminated all 46 genotypes from each other and resulted in definite desi and kabuli cultivar-specific groupings. Most of the desi and kabuli genotypes were grouped in separate clusters (I and II), which further corresponded well with their known pedigree relationships and parentage with slight deviations. However, desi genotypes included under cluster I were further grouped into four different sub-clusters (Ia, Ib, Ic, and Id), while kabuli genotypes belonging to cluster II classified into two different sub-clusters (IIa and IIb; Figure 6).

The population genetic structure among 31 desi and 15 kabuli chickpea genotypes was determined using 160 validated polymorphic SSR markers with varying levels of population numbers $(K=2-10)$ with 20 replications. The optimization of $K$ inferred that at $K=5$, the average estimate of $\operatorname{Ln} \mathrm{P}(\mathrm{D})$ across 20 independent replications plateaus and also best replicate giving maximum log likelihood values with sharp peak was obtained. All 46 chickpea genotypes were majorly classified into two distinct high resolution population groups (Figure 7). The population groups, I (31 desi and one kabuli chickpea) and II (14 kabuli chickpea) contained the genotypes mostly from desi and kabuli chickpea, respectively. The desi population group (I) was further classified into four sub-population groups; Ia (10 


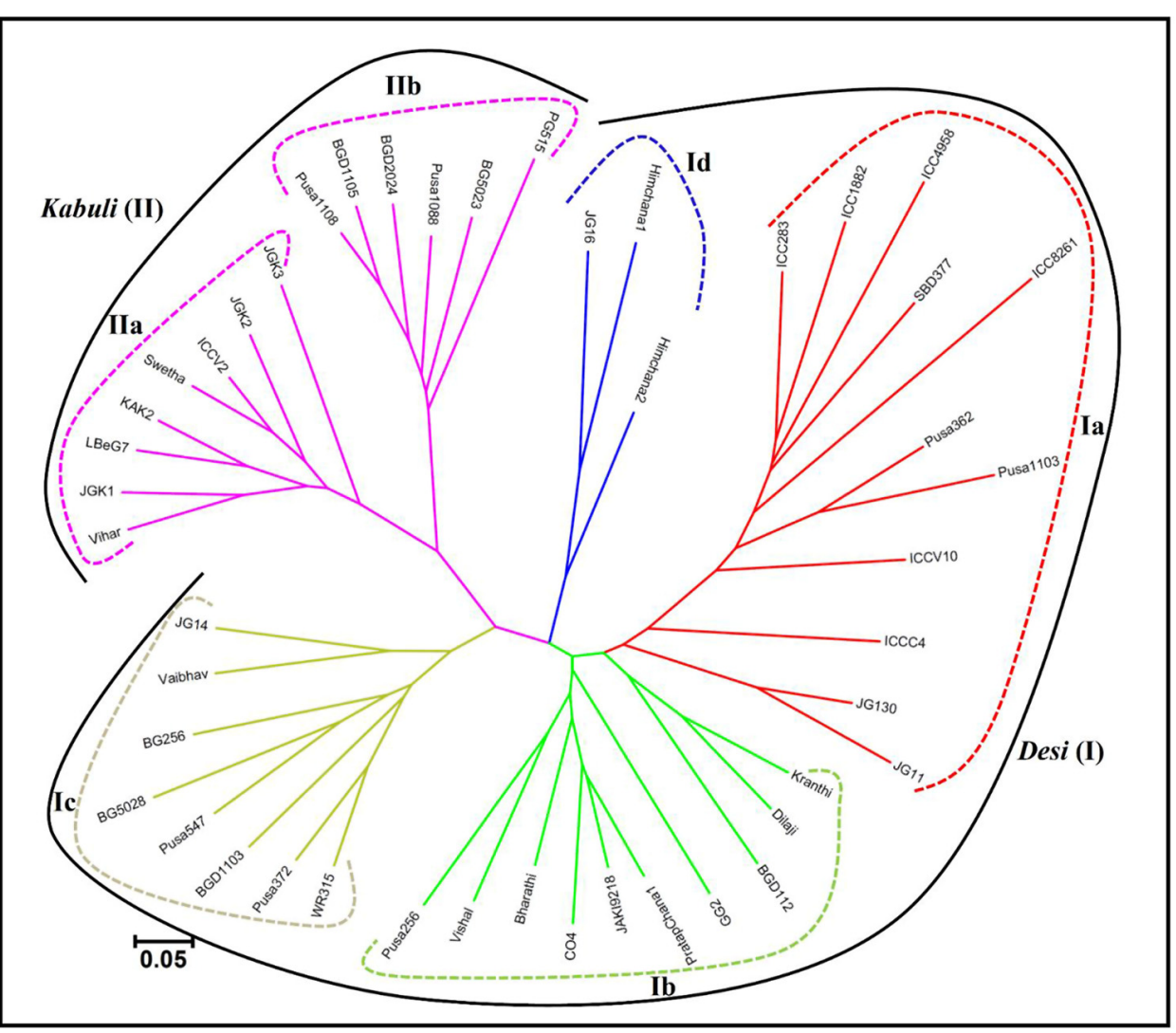

FIGURE 6 | Unrooted phylogenetic tree depicting the genetic relationships among desi and kabuli genotypes. The tree was generated based on Nei's genetic distance using genotyping data of 160 informative SSR markers in 31 desi and 15 kabuli chickpea genotypes. Molecular classification clearly differentiated genotypes into six different clusters, which corresponded to their cultivar-specific origin and parentage/known pedigree relationships.

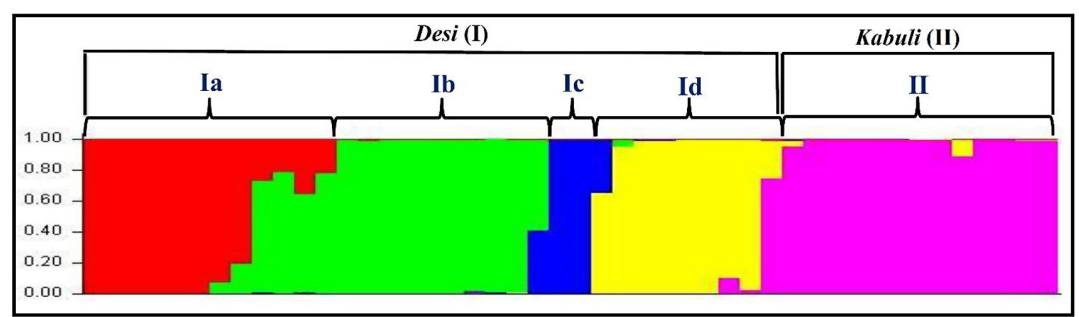

FIGURE 7 | Population genetic structure inferred best possible structure among desi and kabuli chickpea genotypes. The genotyping data of 160 informative genome-wide SSR markers in 31 desi and 15 kabuli chickpea genotypes was used for this analysis. These mapped markers assigned 46 chickpea genotypes into five populations that majorly grouped accordingly by their cultivar-specific origin and parentage/pedigree relationships. The accessions represented by vertical bars along the horizontal axis were classified into $K$ color segments based on their estimated membership fraction in each $K$ cluster. Five diverse colors represent different population groups based on optimal population number $K=5$. desi and one kabuli chickpea genotypes), Ib (10 desi), Ic (8 desi), and Id (3 desi) (Figure 7). The cultivar-specific classification and geographical origin of 46 chickpea genotypes belonging to all the five individual population groups are provided in the Supplementary Table S3. The population groupings obtained among 46 chickpea genotypes corresponded well with their origin and pedigree relationships/parentage. This was further consistent with the clustering patterns and genetic relationships as obtained by the NJ tree analysis. Further, molecular genetic variation among and within five populations was estimated using above 160 informative SSR markers. It revealed a wider level of quantitative genetic differentiation $\left(F_{\mathrm{ST}}\right.$ varied from $0.16-$ 0.91 with an average of 0.64 ) among five population groups. The genetic variation among the five population groups (mean $F_{\text {ST }}$ : 0.62) was higher than that estimated within populations (0.53). Higher molecular diversity of population group I (mean 
$\left.F_{\mathrm{ST}}: 0.86\right)$ as compared to group II $(0.69)$ was evident. Within population groups I and II, maximum divergence was observed in population groups Ib (mean $F_{\mathrm{ST}}$ : 0.83) and IIb (0.65), respectively. All the 46 chickpea genotypes clearly belonged to a structured population of five distinct groups in which about $74.2 \%$ of inferred ancestry of each group was derived from one of the model-based population and remaining $\sim 25.8 \%$ contained admixed ancestry. Maximum admixtures $(20.3 \%)$ of the three desi population groups (Ib, Ic, and Id) with kabuli population (II) were observed.

\section{CMsDB: Features and Utility}

We developed a public data resource, $\mathrm{CMsDB}$, to provide a searchable interface to the SSR data reported in this study. CMsDB is publicly available at http://www.nipgr.res.in/CMsDB. html. The database provides browsable access to all the SSRs identified in desi and kabuli chickpea types, and polymorphic SSRs between them. CMsDB can be used to retrieve SSRs in desi and kabuli genomes using various simple [genomic location (chromosome number and position) and genomic feature (genic and/or inter-genic)] and advanced [motif type (di- to hexa-nucleotide), motif sequence, repeat number and repeat unit length] search parameters. Multiple parameters can be combined also to search for a specific set of SSRs as per user requirement. The output lists all the SSRs meeting the user- selected parameters(s) in tabulated format along with various information, including SSR identifier, chromosome number, motif type and length, genomic location (start and end position in bp) and location in the genomic features (genic/intergenic, gene identifier and intron/exon/upstream sequence). An option for downloading the flanking sequences (50-250 bp) of individual/multiple SSRs has also been provided. Polymorphic SSRs between desi/kabuli can also be searched/retrieved using similar parameters. Further, CMsDB provides information on the primers designed for the polymorphic SSRs. In addition to download the flanking sequences (50-250 bp), an option for viewing/downloading the designed primers for individual/multiple polymorphic SSRs has also been provided. Whole datasets have also been made available for download for high-throughput genotyping applications. We aim to update the database as the new versions of desi and kabuli reference genome sequence data set(s) will become available for chickpea. Figure 8 provides snapshots of various features and utilities of the CMsDB.

\section{Discussion}

The development and large-scale validation of informative genome-wide SSR markers showing high intra-specific

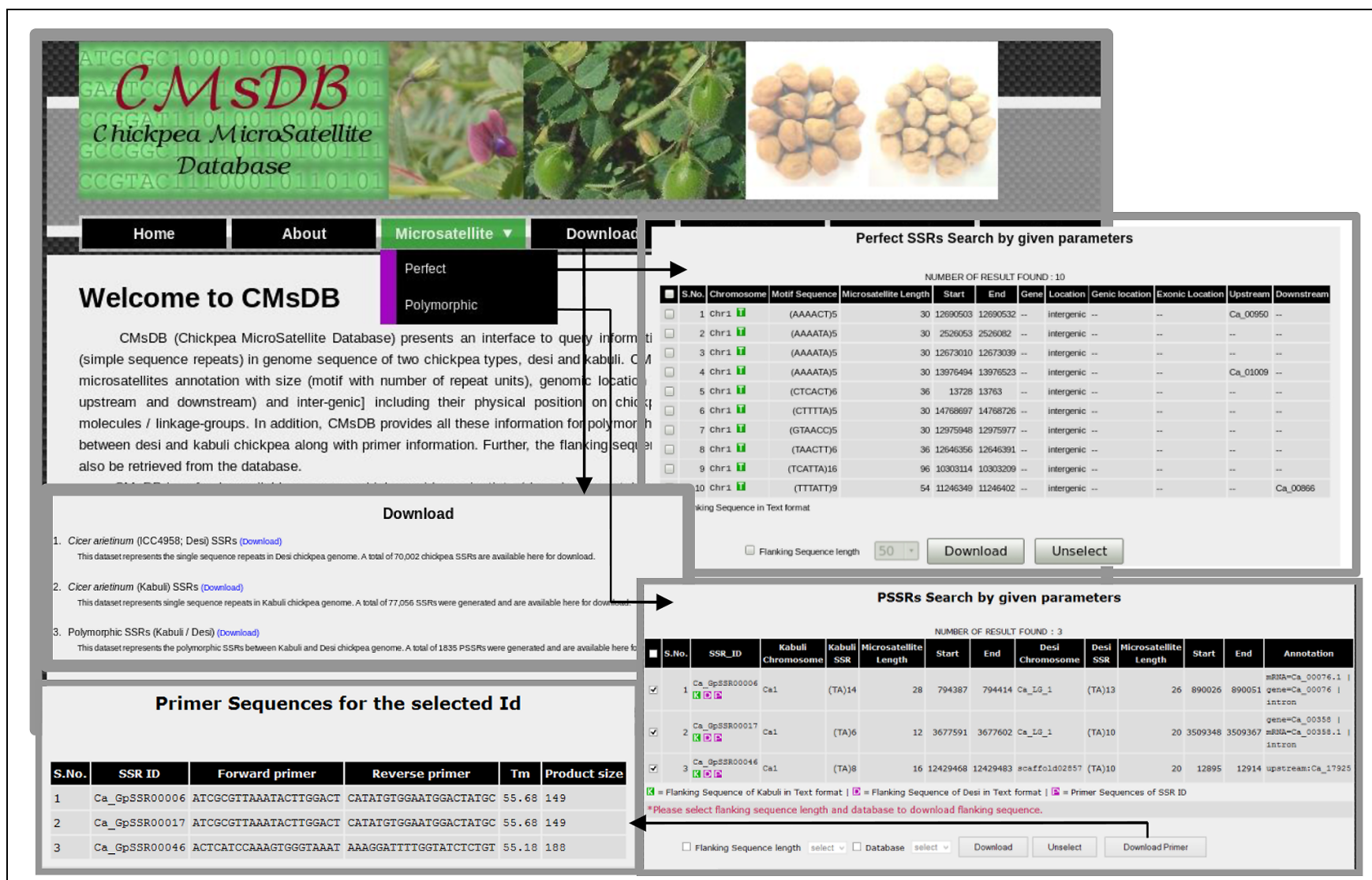

FIGURE 8 | Snapshots of the public web resource CMsDB showing its various utilities. The snapshots were taken from the database webpages. 
polymorphic potential among desi and kabuli genotypes is required for marker-assisted genetic improvement of chickpea. In this context, we discovered 74941 and 81845 SSRs from the desi and kabuli chickpea genomes, respectively, and inferred their frequency and genomic distribution in the intergenic regions and within protein-coding genes. Subsequently, 1835 polymorphic (based on SSR repeat-length variation) SSRs between desi and kabuli genomes from different coding and non-CDS components were identified and characterized. CMsDB provides an integrated web interface to search, browse and filter the SSRs in desi and kabuli chickpea genomes and polymorphic SSRs between them. Recently, the CicArMiSatDB comprising SSR markers identified from coding and non-coding regions of kabuli genome (Varshney et al., 2013) has been constructed (Doddamani et al., 2014). CMsDB developed in our study provides additional interfaces for genome-wide SSR markers from both desi and kabuli chickpea, and polymorphic SSR markers (derived from different coding and non-CDS components) between them, Thus, CMsDB will assist molecular breeders in rapid selection of gene-based polymorphic SSR markers for use in large-scale genotyping applications of chickpea.

Large-scale genome-wide SSR markers (designed from diverse coding, non-coding and intergenic regions) showing polymorphism between desi and kabuli chickpea developed in our study will serve as an immediate resource for mapping whole genome and targeted mapping of traitspecific genes/QTLs for marker-assisted genetic improvement in chickpea. These polymorphic SSR markers being derived from different coding and non-CDS components that regulate cellular and biological functions have significance in developing functional genetic markers for rapidly establishing markertrait linkages and identification of genes/QTLs associated with important agronomic traits. The association of gene-based SSR markers based on their expansion/contraction of repeats with many traits of agricultural importance including seed weight in chickpea have been well studied (Kujur et al., 2013, 2014).

Higher $(\sim 74 \%)$ amplification success rate of polymorphic SSR markers in chickpea genotypes suggested their immense use in various genotyping applications in chickpea. The remaining $\sim 26 \%$ of SSR markers derived mostly from the introns and intergenic regions of genes showed null amplification in chickpea genotypes. It could be due to insertion-deletions in the primer-binding sites of corresponding genomic sequences of desi and kabuli chickpea. This may also result from frequent association of transposable elements with the intronic and intergenic SSRs as previously documented in rice (Temnykh et al., 2001; Parida et al., 2009). The extent of intra-specific polymorphic potential (63.5\%, 2-12 alleles and PIC: 0.75$)$ detected in our study is much higher than that obtained previously using random SSR markers ( $\sim 35-40 \%$; Sethy et al., 2006; Nayak et al., 2010; Bharadwaj et al., 2011; Gujaria et al., 2011; Hiremath et al., 2011; Kujur et al., 2013) and polymorphic SSR markers (50-60\%, Hiremath et al., 2011; Agarwal et al., 2012). The maximum polymorphic potential (average 63\% and mean PIC: 0.69 ) of SSR markers specifically derived from the intergenic, upstream regulatory regions and intronic sequences in chickpea agreed well with the previous reports in crop plants (Grover et al., 2007; Zhang et al., 2007; Parida et al., 2009, 2010). It suggested the utility of polymorphic SSRs derived from non-CDS component of genes in chickpea. The presence of abundant di- and tetra-nucleotide SSR repeats with their specific characteristics of showing high replication slippage than tri-nucleotide SSRs (more constrained by selective pressure), particularly in the upstream regulatory regions, introns and intergenic regions might be contributing to their high polymorphic potential. Among the identified polymorphic genome-wide SSR markers, the class I and noncoding gene sequence-derived SSR markers were found more informative and thus would have greater utility in rapid selection of polymorphic markers for efficient genotyping applications in chickpea. The SSR marker-based polymorphic potential $(64.7 \%$, PIC: 0.75) among desi and kabuli chickpea genotypes was higher (47.6\%, PIC: 0.62 ) than that within desi or kabuli genotypes. Henceforth, the developed genome-wide SSR markers being more informative (in terms of high intra-specific polymorphic potential as well as functional significance) than SSR markers identified till now would be of immediate use in efficient large-scale genotyping applications in chickpea. Considering requirement of functional SSR markers showing high intraspecific polymorphism among desi and kabuli genotypes for chickpea marker-assisted genetic enhancement, the large-scale experimentally validated polymorphic SSR markers developed by us will be highly relevant.

A wider level of genetic differentiation $\left(F_{\mathrm{ST}}\right.$ varied from 0.16 to 0.94 with a mean of 0.68 ) obtained among 46 chickpea genotypes belonging to five population groups was comparable/higher than the previously detected level (0.030.82) with the genomic and genic SSR markers (Sethy et al., 2006; Choudhary et al., 2009; Bharadwaj et al., 2011; Kujur et al., 2013). The higher molecular diversity between desi and kabuli population groups than that obtained within desi and kabuli populations is expected in a self-pollinated crop species like chickpea. Higher genetic differentiation within desi population in contrast to kabuli agreed well with earlier observations (Upadhyaya et al., 2008; Bharadwaj et al., 2011; Kujur et al., 2013). Therefore, wider molecular diversity and genetic base detected by genome-wide informative SSR markers would be much relevant in the selection of desirable plant types for varietal improvement in chickpea. The admixed ancestry ( $\sim 25.8 \%$ ) among six populations might be due to their complex breeding history involving inter-crossing and introgression among desi and kabuli chickpea genotypes along with strong selection pressure and evolutionary bottlenecks during chickpea domestication. Maximum admixtures and close phylogenetic relationships between desi and kabuli populations is consistent with the earlier morphological, cytological and biochemical documentation and molecular studies using SSR markers (Abbo et al., 2003; Berger et al., 2003; Sethy et al., 2006; Upadhyaya et al., 2008; Bharadwaj et al., 2011; Jhanwar et al., 2012; Kujur et al., 2013). It is also supported with the commonly accepted presumption related to origination and domestication of desi and kabuli chickpea at archeological sites of South Eastern Turkey nearly about 10000 years ago (Abbo et al., 2003; Berger et al., 2003). 
The distinctness and phylogenetic relationships established by informative genome-wide SSR markers in desi and kabuli chickpea genotypes belonging to five population groups are in accordance with their cultivar-specific origin and parentage/pedigree relationships. For instance, the genotypes classified under desi (31) and kabuli (15) population groups had distinct agro-morphological features that are commonly observed in desi (purple flower and small seed size with yellow brown to light brown colored seed coat) and kabuli (white flower and large seed size with beige colored seed coat) chickpea, respectively. The grouping of one kabuli chickpea genotype ICC 8261 (originated from Turkey) with the desi genotypes (originated from India) of population group Ia reflected more influence of its geographical origin rather than cultivar-specific classification. However, complex breeding history involving introgression, cross-breeding efforts and sequential evolutionary bottlenecks among desi genotypes possibly led to their clustering in four population sub-groups. The informative genome-wide SSR markers developed by us are significant in establishing distinctness and evolutionary relationships as well as assaying broader molecular diversity among desi and kabuli chickpea genotypes and therefore, will be useful for many applications in chickpea genetics, genomics and breeding.

\section{Conclusion}

We developed a large set of polymorphic SSR markers between desi and kabuli chickpea from different coding

\section{References}

Abbo, S., Berger, J., and Turner, N. C. (2003). Evolution of cultivated chickpea: four bottlenecks limit diversity and constrain adaptation. Funct. Plant Biol. 30, 1081-1087. doi: 10.1071/FP03084

Abbo, S., Molina, C., Jungmann, R., Grusak, M. A., Berkovitch, Z., Reifen, R., et al. (2005). Quantitative trait loci governing carotenoid concentration and weight in seeds of chickpea (Cicer arietinum L.). Theor. Appl. Genet. 111, 185-195. doi: 10.1007/s00122-005-1930-y

Agarwal, G., Jhanwar, S., Priya, P., Singh, V. K., Saxena, M. S., Parida, S. K., et al. (2012). Comparative analysis of kabuli chickpea transcriptome with desi and wild chickpea provides a rich resource for development of functional markers. PLoS ONE 7:e52443. doi: 10.1371/journal.pone.0052443

Anuradha, C., Gaur, P. M., Pande, S., Gali, K. K., Ganesh, G., Kumar, J., et al. (2011). Mapping QTL for resistance to Botrytis grey mould in chickpea. Euphytica 182, 1-9. doi: 10.1007/s10681-011-0394-1

Bao, J. S., Corke, H., and Sun, M. (2002). Microsatellites in starch-synthesizing genes in relation to starch physicochemical properties in waxy rice (Oryza sativa L.). Theor. Appl. Genet. 105, 898-905. doi: 10.1007/s00122-0021049-3

Berger, J., Abbo, S., and Turner, N. C. (2003). Ecogeography of annual wild Cicer species: the poor state of the world collection. Crop Sci. 43, 1076-1090. doi: 10.2135/cropsci2003.1076

Bharadwaj, C., Srivastava, R., Chauhan, S. K., Satyavathi, C. T., Kumar, J., Faruqui A., et al. (2011). Molecular diversity and phylogeny in geographical collection of chickpea (Cicer sp.) accessions. J. Genet. 90, e94-e100.

Chabane, K., Ablett, G. A., Cordeiro, G. M., Valkoun, J., and Henry, R. J. (2005). EST versus genomic derived microsatellite markers for genotyping wild and cultivated barley. Genet. Resour. Crop Evol. 52, 903-909. doi: 10.1007/s10722003-6112-7 and non-CDS components of genes and intergenic regions. These genome-wide physically mapped markers with relatively high experimental validation success rate and intra-/interspecific polymorphic potential have immense utility in largescale genotyping applications in chickpea. A wider molecular (functional) diversity including parentage- and cultivar-specific phylogenetic relationships assayed by these informative SSR markers in a structured desi and kabuli population suggested their significance in chickpea structural, functional and comparative genomics, and breeding. We anticipate that web resource $\mathrm{CMsDB}$ will be very useful to scientists/breeders to search, browse and query SSRs in chickpea to facilitate molecular breeding strategies in chickpea.

\section{Acknowledgments}

This work was financially supported by the core grant from NIPGR and the Department of Biotechnology, Government of India, New Delhi, under the Next Generation Challenge Programme on Chickpea Genomics (grant number BT/PR12919/AGR/02/676/2009 from 2009 to 2014). RG acknowledges the INSPIRE Faculty Award from the Department of Science and Technology, Government of India.

\section{Supplementary Material}

The Supplementary Material for this article can be found online at: http://journal.frontiersin.org/article/10.3389/fpls.2015.00645

Cho, Y. G., Ishii, T., Temnykh, S., Chen, X., Lipovich, L., McCouch S. R., et al. (2000). Diversity of microsatellites derived from genomic libraries and GenBank sequences in rice (Oryza sativa L.). Theor. Appl. Genet. 100, 713-722. doi: $10.1007 /$ s001220051343

Choudhary, S., Sethy, N. K., Shokeen, B., and Bhatia, S. (2009). Development of chickpea EST-SSR markers and analysis of allelic variation across related species. Theor. Appl. Genet. 118, 591-608. doi: 10.1007/s00122-008-0923-z

Deokar, A. A., Ramsay, L., Sharpe, A. G., Diapari, M., Sindhu, A., Bett, K., et al. (2014). Genome wide SNP identification in chickpea for use in development of a high density genetic map and improvement of chickpea reference genome assembly. BMC Genomics 15:708. doi: 10.1186/1471-2164-15-708

Doddamani, D., Katta, M. A., Khan, A. W., Agarwal, G., Shah, T. M., and Varshney, R. K. (2014). CicArMiSatDB: the chickpea microsatellite database. BMC Bioinformatics 15:212. doi: 10.1186/1471-2105-15-212

Dresselhaus, T., Cordts, S., Heuer, S., Sauter, M., Lörz, H., and Kranz, E. (1999). Novel ribosomal genes from maize are differentially expressed in the zygotic and somatic cell cycles. Mol. Gen. Genet. 261, 416-427. doi: $10.1007 / \mathrm{s} 004380050983$

Fujimori, S., Washio, T., Higo, K., Ohtomo, Y., Murakami, K., Matsubara, K., et al. (2003). A novel feature of microsatellites in plants: a distribution gradient along the direction of transcription. FEBS Lett. 554, 17-22. doi: 10.1016/S00145793(03)01041-X

Garg, R., Patel, R. K., Jhanwar, S., Priya, P., Bhattacharjee, A., Yadav, G., et al. (2011). Gene discovery and tissue-specific transcriptome analysis in chickpea with massively parallel pyrosequencing and web resource development. Plant Physiol. 156, 1661-1678. doi: 10.1104/pp.111.178616

Gaur, R., Azam, S., Jeena, G., Khan, A. W., Choudhary, S., Jain, M., et al. (2012). High-throughput SNP discovery and genotyping for constructing a saturated linkage map of chickpea (Cicer arietinum L.). DNA Res. 19, 357-373. doi: $10.1093 /$ dnares/dss018 
Grover, A., Aishwarya, V., and Sharma, P. C. (2007). Biased distribution of microsatellite motifs in the rice genome. Mol. Genet. Genomics 277, 469-480. doi: 10.1007/s00438-006-0204-y

Gujaria, N., Kumar, A., Dauthal, P., Dubey, A., Hiremath, P., Prakash A. B., et al. (2011). Development and use of genic molecular markers (GMMs) for construction of a transcript map of chickpea (Cicer arietinum L.). Theor. Appl. Genet. 122, 1577-1589. doi: 10.1007/s00122-011-1556-1

Hiremath, P. J., Farmer, A., Cannon, S. B., Woodward, J., Kudapa, H., Tuteja, R., et al. (2011). Large-scale transcriptome analysis in chickpea (Cicer arietinum L.), an orphan legume crop of the semi-arid tropics of Asia and Africa. Plant Biotechnol. J. 9, 922-931. doi: 10.1111/j.1467-7652.2011.00625.x

Hiremath, P. J., Kumar, A., Penmetsa, R. V., Farmer, A., Schlueter, J. A., Chamarthi, S. K., et al. (2012). Large-scale development of cost-effective SNP marker assays for diversity assessment and genetic mapping in chickpea and comparative mapping in legumes. Plant Biotechnol. J. 10, 716-732. doi: 10.1111/j.14677652.2012.00710.x

Jaganathan, D., Thudi, M., Kale, S., Azam, S., Roorkiwal, M., Gaur, P. M., et al. (2014). Genotyping-by-sequencing based intra-specific genetic map refines a "QTL-hotspot" region for drought tolerance in chickpea. Mol. Genet. Genomics 290, 559-571. doi: 10.1007/s00438-014-0932-3

Jain, M., Misra, G., Patel, R. K., Priya, P., Jhanwar, S., Khan, A. W., et al. (2013). A draft genome sequence of the pulse crop chickpea (Cicer arietinum L.). Plant J. 74, 715-729. doi: 10.1111/tpj.12173

Jhanwar, S., Priya, P., Garg, R., Parida, S. K., Tyagi, A. K., and Jain, M. (2012). Transcriptome sequencing of wild chickpea as a rich resource for marker development. Plant Biotechnol. J. 10, 690-702. doi: 10.1111/j.14677652.2012.00712.x

Kujur, A., Bajaj, D., Saxena, M. S., Tripathi, S., Upadhyaya, H. D., Gowda, C. L., et al. (2013). Functionally relevant microsatellite markers from chickpea transcription factor genes for efficient genotyping applications and trait association mapping. DNA Res. 20, 355-374. doi: 10.1093/dnares/dst015

Kujur, A., Bajaj, D., Saxena, M. S., Tripathi, S., Upadhyaya, H. D., Gowda, C. L. L., et al. (2014). An efficient and cost-effective approach for genic microsatellite marker-based large-scale trait association mapping: identification of candidate genes for seed weight in chickpea. Mol. Breed. 34, 241-265. doi: 10.1007/s11032014-0033-3

Lawson, M. J., and Zhang, L. (2006). Distinct patterns of SSR distribution in the Arabidopsis thaliana and rice genome. Genome Biol. 7, R14. doi: 10.1186/gb2006-7-2-r14

Li, Y. C., Korol, A. B., Fahima, T., and Nevo, E. (2004). Microsatellites within genes: structure, function, and evolution. Mol. Biol. Evol. 21, 991-1007. doi: 10.1093/molbev/msh073

Liu, K., and Muse, S. V. (2005). PowerMarker: an integrated analysis environment for genetic marker analysis. Bioinformatics 21, 2128-2129. doi: 10.1093/bioinformatics/bti282

Millan, T., Winter, P., Jungling, R., Gil, J., Rubio, J., Cho, S., et al. (2010). A consensus genetic map of chickpea (Cicer arietinum L.) based on 10 mapping populations. Euphytica 175, 175-189. doi: 10.1007/s10681-010-0157-4

Nayak, S. N., Zhu, H., Varghese, N., Datta, S., Choi, H. K., Horres, R., et al. (2010). Integration of novel SSR and gene-based SNP marker loci in the chickpea genetic map and establishment of new anchor points with Medicago truncatula genome. Theor. Appl. Genet. 120, 1415-1441. doi: 10.1007/s00122-010-1265-1

Nei, M., Tajima, F., and Tateno, Y. (1983). Accuracy of estimated phylogenetic trees from molecular data. J. Mol. Evol. 19, 153-170. doi: 10.1007/BF02300753

Parida, S. K., Dalal, V., Singh, N. K., and Mohapatra, T. (2009). Genic non-coding microsatellites in the rice genome: characterization, marker design and use in assessing genetic and evolutionary relationships among domesticated groups. BMC Genomics 10:140. doi: 10.1186/1471-2164-10-140

Parida, S. K., RajKumar, K. A., Dalal, V., Singh, N. K., and Mohapatra, T. (2006). Unigene derived microsatellite markers for the cereal genomes. Theor. Appl. Genet. 112, 808-817. doi: 10.1007/s00122-005-0182-1

Parida, S. K., Yadava, D. K., and Mohapatra, T. (2010). Microsatellites in Brassica unigenes: relative abundance, marker design and use in comparative physical mapping and genome analysis. Genome 53, 55-67. doi: 10.1139/g09-084

Pritchard, J. K., Stephens, M., and Donnelly, P. (2000). Inference of population structure using multilocus genotype data. Genetics 155, 945-959.

Radhika, P., Gowda, S. J., Kadoo, N. Y., Mhase, L. B., Jamadagni, B. M., Sainani, M. N., et al. (2007). Development of an integrated intra-specific map of chickpea
(Cicer arietinum L.) using two recombinant inbred line populations. Theor. Appl. Genet. 115, 209-216. doi: 10.1007/s00122-007-0556-7

Roorkiwal, M., Sawargaonkar, S. L., Chitikineni, A., Thudi, M., Saxena, R. K., Upadhyaya, H. D., et al. (2013). Single nucleotide polymorphism genotyping for breeding and genetics applications in chickpea and pigeonpea using the BeadXpress platform. Plant Genome 6, 1-10. doi: 10.3835/plantgenome2013.05.0017

Sethy, N. K., Shokeen, B., Edwards, K. J., and Bhatia, S. (2006). Development of microsatellite markers and analysis of intra-specific genetic variability in chickpea (Cicer arietinum L.). Theor. Appl. Genet. 112, 1416-1428. doi: 10.1007/s00122-006-0243-0

Temnykh, S., DeClerck, G., Lukashova, A., Lipovich, L., Cartinhour, S., and McCouch, S. (2001). Computational and experimental analysis of microsatellites in rice (Oryza sativa L.): frequency, length-variation, transposon associations and genetic marker potential. Genome Res. 11, 1441-1452. doi: 10.1101/gr.184001

Thudi, M., Bohra, A., Nayak, S. N., Varghese, N., Shah, T. M., Penmetsa, R. V., et al. (2011). Novel SSR markers from BAC-end sequences, DArT arrays and a comprehensive genetic map with 1,291 marker loci for chickpea (Cicer arietinum L.). PLoS ONE 6:e27275. doi: 10.1371/journal.pone.0027275

Upadhyaya, H. D., Dwivedi, S. L., Baum, M., Varshney, R. K., Udapa, S. M., Gowda, C. L., et al. (2008). Genetic structure, diversity, and allelic richness in composite collection and reference set in chickpea (Cicer arietinum L.). BMC Plant Biol. 8:106. doi: 10.1186/1471-2229-8-106

Vadez, V., Krishnamurthy, L., Thudi, M., Anuradha, C., Colmer, T. D., Turner, N. C., et al. (2012). Assessment of ICCV $2 \times$ JG 62 chickpea progenies shows sensitivity of reproduction to salt stress and reveals QTLs for seed yield and seed number. Mol. Breed. 30, 9-21. doi: 10.1007/s11032-011-9594-6

Varshney, R. K., Graner, A., and Sorrells, M. E. (2005). Genic microsatellite markers in plants: features and applications. Trends Biotechnol. 23, 48-55. doi: 10.1016/j.tibtech.2004.11.005

Varshney, R. K., Song, C., Saxena, R. K., Azam, S., Yu, S., Sharpe, A. G., et al. (2013). Draft genome sequence of chickpea (Cicer arietinum) provides a resource for trait improvement. Nat. Biotechnol. 31, 240-246. doi: 10.1038/nbt.2491

Varshney, R. K., Thudi, M., Nayak, S. N., Gaur, P. M., Kashiwagi, J., Krishnamurthy, L., et al. (2014). Genetic dissection of drought tolerance in chickpea (Cicer arietinum L.). Theor. Appl. Genet. 127, 445-462. doi: 10.1007/s00122-013-2230-6

Winter, P., Benko-Iseppon, A. M., Huttel, B., Ratnaparkhe, M., Tullu, A., Sonnante, G., et al. (2000). A linkage map of the chickpea (Cicer arietinum L.) genome based on the recombinant inbred lines from a C. arietinum $\mathrm{x}$ C. reticulatum cross, localization of resistance genes for Fusarium races 4 and 5. Theor. Appl. Genet. 101, 1155-1163. doi: 10.1007/s001220051592

Yu, J., Hu, S. N., Wang, J., Wong, G. K., Li, S., Liu, B., et al. (2002). A draft sequence of the rice genome (Oryza sativa L. ssp. indica). Science 296, 79-92. doi: 10.1126/science.1068037

Zhang, L., Zuo, K., Zhang, F., Cao, Y., Wang, J., Zhang, Y., et al. (2006). Conservation of noncoding microsatellites in plants: implication for gene regulation. BMC Genomics 7:323. doi: 10.1186/1471-2164-7-323

Zhang, S., Tang, C., Zhao, Q., Li, J., Yang, L., Qie, L., et al. (2014). Development of highly polymorphic simple sequence repeat markers using genome-wide microsatellite variant analysis in foxtail millet [Setaria italica (L.) P. Beauv] BMC Genomics 15:78. doi: 10.1186/1471-2164-15-78

Zhang, Z., Deng, Y., Tan, J., Hu, S., Yu, J., and Xue, Q. (2007). A genome-wide microsatellite polymorphism database for the indica and japonica rice. DNA Res. 14, 37-45. doi: 10.1093/dnares/dsm005

Conflict of Interest Statement: The authors declare that the research was conducted in the absence of any commercial or financial relationships that could be construed as a potential conflict of interest.

Copyright (c) 2015 Parida, Verma, Yadav, Ambawat, Das, Garg and Jain. This is an open-access article distributed under the terms of the Creative Commons Attribution License (CC BY). The use, distribution or reproduction in other forums is permitted, provided the original author(s) or licensor are credited and that the original publication in this journal is cited, in accordance with accepted academic practice. No use, distribution or reproduction is permitted which does not comply with these terms. 